\title{
REVIEW
}

\section{The nucleolus: functional organization and assembly}

\author{
Danièle Hernandez-Verdun \\ Institut Jacques Monod, Paris, France
}

Received $23^{\text {rd }}$ May 2003.

Published online $4^{\text {th }}$ July 2003.

\begin{abstract}
Summary
The nucleolus is a large nuclear domain generated by the act of building ribosomes. It illustrates the compartmentation of the nuclear functions, since it is in the nucleolus that transcription of the ribosomal genes, maturation and processing of the 47S ribosomal RNAs (rRNAs) into $18 \mathrm{~S}, 5.8 \mathrm{~S}$ and $28 \mathrm{~S}$ rRNA, and almost complete assembly of the $40 \mathrm{~S}$ and $60 \mathrm{~S}$ ribosome subunits take place. The shape, size and organization of the nucleoli vary with their activity. Nuleolar activity is a cell cycle dependent-process. In electron microscopy, the nucleolus exhibits three main components: fibrillar centers (FCs), a dense fibrillar component (DFC) and a granular component (GC), corresponding to different steps of ribosome biogenesis. The steady state between transcription, processing and export of ribosomal subunits engenders this organization. Conversely, inactivation or blockage of one of these processes modifies the organization of the nucleolus and ultimately induces nucleolar disassembly. The nucleolus is also a plurifunctional domain, a key partner of chromatin architecture in the nucleus and it plays a crucial role in several cellular functions in addition to ribosome production.

The nucleolus is assembled at the end of mitosis, is active during interphase, and disassembled in prophase. The nucleolar transcription and processing machineries are inherited from parental to daughter cells through mitosis. The polymerase I (pol I)_transcription machinery is repressed during mitosis although assembled with ribosomal genes. Repression of pol I transcription is achieved at the end of prophase and is maintained during mitosis through phosphorylation of transcription factors by the cyclin-dependent kinase (CDK) 1 . The nucleolar processing machineries relocalize from the nucleolus towards the periphery of all chromosomes until telophase and this chromosome association depends on CDK1 activity. As a consequence of natural inhibition of CDK1 activity, pol I transcription is restored in telophase. The processing machineries are recruited to the sites of rDNA transcription after a temporary transit in foci known as prenucleolar bodies.

In conclusion, the behavior of the nucleolus illustrates the fact that the dynamics of nuclear organization are integrated in a network of interactions and controls that is largely dependent on the coordination of cell cycle controls.
\end{abstract}

Keywords: nucleolus - cell cycle - dynamics - organization - nuclear domain - ribosome

\section{INTRODUCTION}

General remarks, definitions

The prominent and refractive structures observed in cell nuclei by phase-contrast microscopy, are nucleoli. During the cell cycle, nucleoli develop from chromosomal sites, called nucleolar organizer regions (NORs). NORs were first described by McClintock in Zea mays in 1934 (McClintock 1934), and have since been shown to contain tandemly arranged genes of ribosomal RNA (rDNA) 


\section{Hernandez-Verdun}

and specific NOR proteins. The nucleoli are nuclear domains devoted to ribosome biogenesis. They contain specialized units for various steps of ribosomal RNA (rRNA) synthesis and units for the maturation and processing of rRNA. The nucleolus is not a stable organelle. Its structure, size and organization depend on ribosome biogenesis. The production of ribosomes is directly correlated with protein synthesis and thus nucleolar morphology varies according to cell type, cell differentiation and the stage of the cell cycle. Various pathologies also affect nucleolar morphology in relation to cell growth and proliferation.
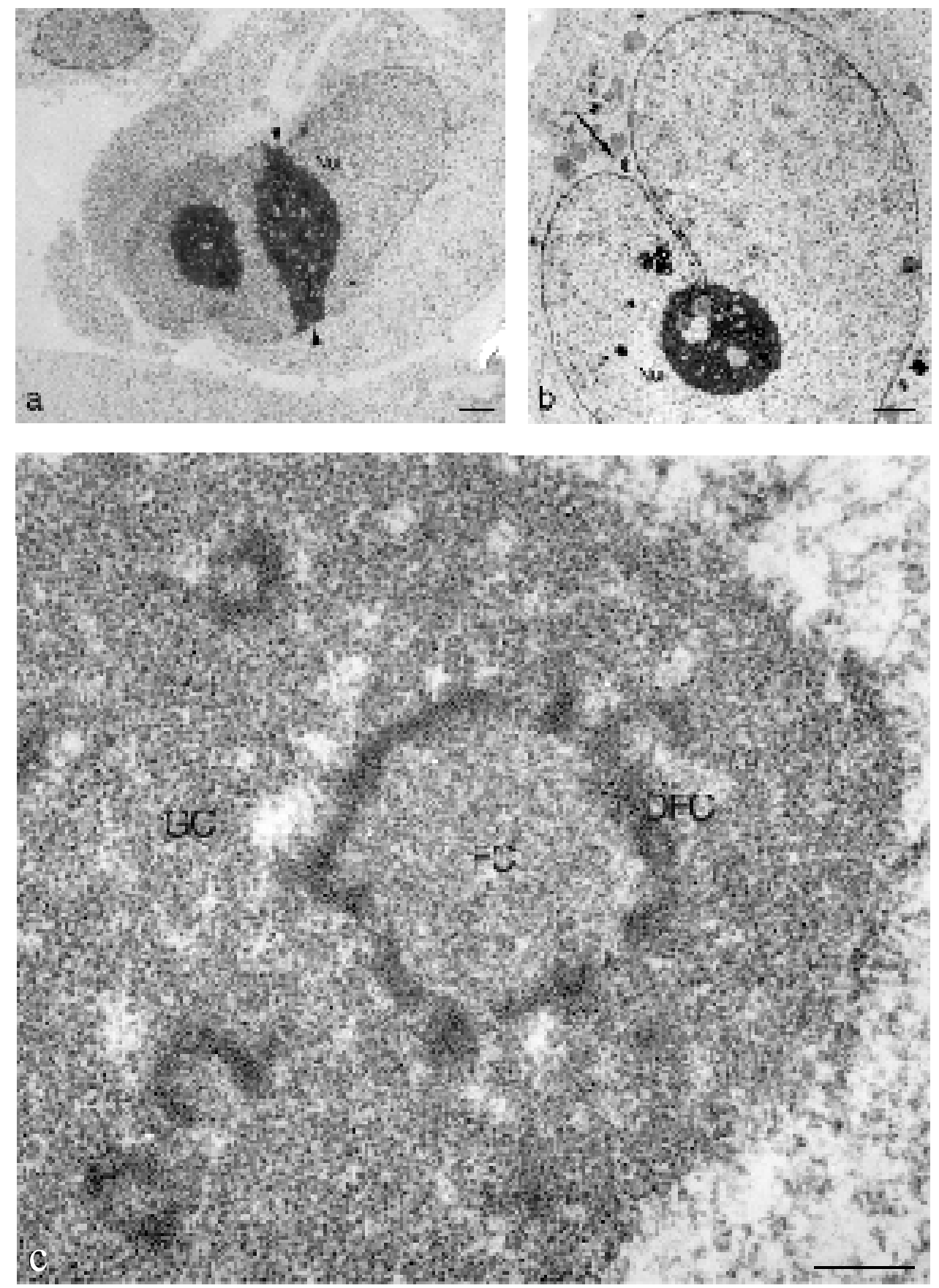

Fig. 1. EM organization of the nucleolus with active ribosome biogenesis. $a$ - General view of HeLa cell in which two nucleoli $(\mathrm{Nu})$ are visible. Note the large size of the nucleoli and the contact with the nuclear envelope (arrow head). Bar: $1 \mu \mathrm{m} ; b$ - The nuclear envelope forms a canal (arrow) which is in contact with the nucleolus ( $\mathrm{Nu}$ ) located in the center of the nucleus. Bar: $1 \mu \mathrm{m} ; c-$ The three nucleolar components are visible: fibrillar center (FC), dense fibrillar component (DFC) and granular component (GC). Bar: $0.5 \mu \mathrm{m}$

Known for several decades as the ribosome factory, the nucleolus was recently proven to be also involved in many other functions (reviewed in (Carmo-Fonseca et al. 2000, Olson et al. 2000, 
Pederson 1998, Visintin and Amon 2000)). Indeed, the nucleolus is also the assembly or processing site for RNA and protein complexes different from rRNA such as the signal recognition particle, tRNA, U6 and RNA-telomerase complex. In addition, it plays a role in the control of the cell cycle by retention of molecules that interact with cell cycle regulators in the nucleoplasm (Tsai and McKay 2002). Furthermore the nucleolus participates in intranuclear traffic between different nuclear domains (Leung and Lamond 2002) and is involved in the general architecture of chromatin in the nucleus (Chubb et al. 2002). From these studies, an interesting concept is emerging that makes the nucleolus a key partner in nuclear architecture playing a crucial role in several cellular functions in addition to ribosome production.

This article will focus on the organization of nucleoli in mammalian cells and on macromolecular aspects of the nucleolar factory. We review information on where, when and how the different steps of ribosome biogenesis are organized in the nucleoli. For more detailed information many reviews are available concerning the functional organization of the nucleolus among them these selected references: (Busch and Smetana 1974, Goessens 1984, Shaw and Jordan 1995), and the exhaustive overview of the subject by Hadjiolov (Hadjiolov 1985).

\section{ORGANIZATION OF THE NUCLEOLUS IN HUMAN CELLS}

Number, size and polarity

Mammalian nuclei contain a small number of nucleoli (Fig. 1 a, b). The number of nucleoli in HeLa cells is between 1 and 6 with considerable variation in size between them. However, the number of nucleoli cannot be an accurate determination since nucleoli associate in human cells after entry into interphase. This phenomenon occurs rapidly (in a few minutes) according to time-lapse recordings in living cells (Savino et al. 2001). Nucleoli vary in form, staining capacity and behavior rendering their classification difficult by light microscopy alone. The shape of nucleoli can differ markedly from one cell type to another, and even within a single cell. In human cells, the diameter of nucleoli varies from less than $1 \mu \mathrm{m}$ in mature lymphocytes to $3-9 \mu \mathrm{m}$ in proliferating cells.

Nucleoli are heavily involved in nuclear polarity. They are traditional cytological landmarks, and there is also evidence of polarity between different nucleoli, between nucleoli and the nuclear envelope and between nucleoli and centromeres.
In the metaphasic plate, the human NOR-bearing chromosomes are closer to each other than would be expected if chromosomal distribution were random. This observation illustrates the polarity of the chromosomes during mitosis, a polarity that is maintained in the interphase nuclei of the two daughter cells as recently demonstrated in living cells (Gerlich et al. 2003). After fusion or in species without nucleolar fusion, the position of nucleoli remains fairly stable, even in rotating nuclei.

In eukaryotic cells nucleoli are very frequently located at or near the nuclear envelope (Fig. 1a). In nuclei with centrally located nucleoli, the nuclear envelope is folded to form the nucleolar canal that is in direct contact with the nucleoli (Fig. 1b). This position seems to be related to the presence of specific skeletal structures at the site of nucleolar attachment to the envelope.

\section{Fine structure}

The fine structure of nucleoli is clearly visualized only by electron microscopic observation of thin sections. In transmission electron microscopy (EM), nucleoli appear to be mainly composed of fibrils and granules (Fig. 1c). The granules are about $150 \mathrm{~nm}$ thick and constitute the granular component (GC) of nucleoli. The fibrils display two different electronopacities. Some fibrils are very contrasted and have been called the dense fibrillar component (DFC), whereas others constitute light areas that have been called fibrillar centers (FCs). Most often, FCs are surrounded by the DFC which is in close contact with the GC (Fig. 1c). Analysis of nucleolar chromatin by specific DNA staining indicates that decondensed chromatin is present in the FC of nucleoli. Fibrillar centers contain not only dispersed chromatin but may also possess clumps or clusters of the condensed chromatin (see e.g. ring shaped nucleoli in Busch and Smetana, 1970). The perinucleolar condensed chromatin, which is more or less abundant depending on the cell type is frequently seen in continuity with the condensed chromatin lining the nuclear envelope.

The relationship between the 3 major nucleolar components was reconstructed using serial thin sections and computer assistance. In PtK1 cells, FCs form discrete structures (about 10 in G1 and 20 in G2) located in different levels throughout the nucleoli and connected by a network of DFC (Junéra et al. 1995). From other 3-D reconstructions, it has become apparent that nucleoli of different cell types exhibit a variable number of FCs of variable sizes, with an inverse proportion between size and number (Hozak et al. 1989, Pébusque and Seïte 1981). 


\section{Mapping ribosome biogenesis in nucleoli}

During the past decades, general mapping of ribosome biogenesis has been achieved in these morphologically distinct nucleolar components. The rDNA sequences were found in the FCs but mainly in the periphery (Puvion-Dutilleul et al. 1997, Thiry and Thiry-Blaise 1991). After short BrUTP incorporation, the nascent transcripts appeared in the junction region between the FCs and the DFC (Puvion-Dutilleul et al. 1997, Shaw and Jordan 1995). The factors of the transcription machinery were detected either in the FCs (Scheer et al. 1984) or in the DFC (Biggiogera et al. 2001, Cmarko et al. 2000). These differences are probably due to the inherent difficulty there is in defining the border zone since discrimination between FC and DFC is based on contrasts that can be modified by the labeling procedure. At present, it is generally accepted that the initiation of rDNA transcription occurs at the junction FC-DFC.

As for processing of the $47 \mathrm{~S}$ rRNA, it starts at the site of transcription in the DFC and continues during the intranucleolar migration of the RNA towards the GC. In the nucleoli, the vectorial distribution of the machineries successively involves correlates with different processing steps, for example, fibrillarin and nucleolin that participate in the early stages of rRNA processing, localize in the
DFC along with the U3 snoRNA (Puvion-Dutilleul et al. 1991), whereas proteins B23 and Nop52 that are involved in intermediate or later stages of ribosome biogenesis localize in the GC. The maturation and processing events, are presently better characterized in yeast (Fatica and Tollervey 2002) than in higher eukaryotes and a very complex process was recently unraveled (Harnpicharnchai et al. 2001). About 50 non-ribosomal proteins are associated with the early nucleolar pre-60S (Nissan et al. 2002). During subsequent maturation these factors are removed and replaced by other factors. Interestingly, the very last step of processing occurs during nucleocytoplasmic transport and finally in the cytoplasm (Nissan et al. 2002).

The confinement of certain machineries in the FC, DFC or GC make it possible to reveal these subnucleolar constituents by immunofluorescence as illustrated for FC, DFC, and GC in figures 3, 4, 5 respectively. These labeling patterns (FC, DFC, DFC) in the nucleoli provide a good indication of the steps of ribosome biogenesis concerned. To the question of what normally maintains a nucleolus in its usual shape and structure, results from different investigators have provided evidence that transient association of functionally related components is necessary to generate a morphologically defined nucleolus with its three distinct components.
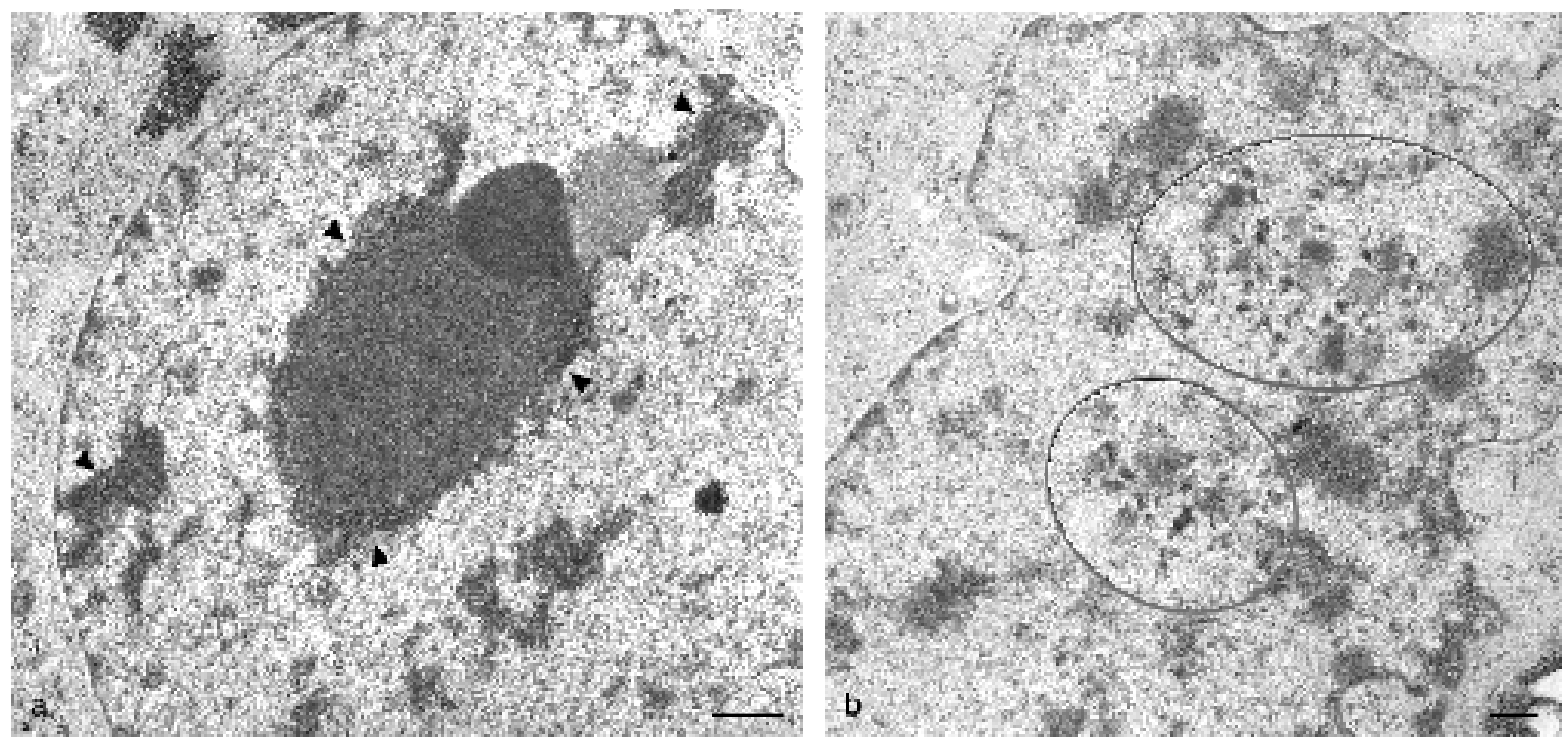

Fig. 2. EM organization of the nucleolus without ribosome biogenesis. a - The segregation of the nucleolar components $2 \mathrm{~h}$ after actinomycine D (AMD) inhibition of rDNA transcription. Note the condensed chromatin visible around the nucleolus and in the nucleoplasm (arrow heads). Bar: $1 \mu \mathrm{m} ; \mathrm{b}$ - Nucleolar disorganization induced by Roscovitine, a CDK inhibitor. The circles indicate the location of the dispersed nucleolar component. Bar: 

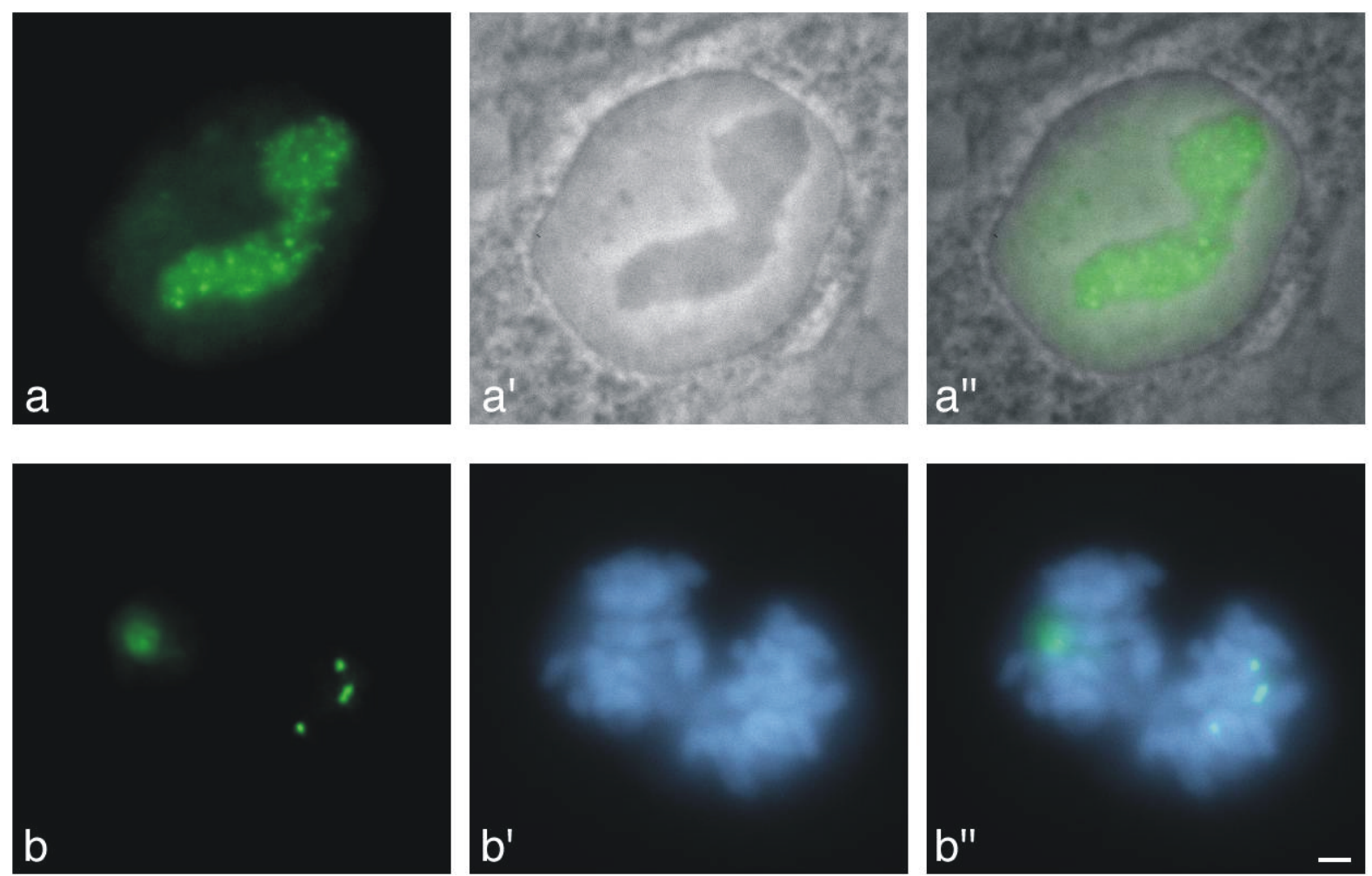

Fig. 3. Localization of rDNA transcription factors. $a$ - Interphasic HeLa cell labeled with anti-UBF antibodies. The localization of UBF is visible in green (FITC) as small foci in the central region of the nucleolus. $a$ ' Same cell observed in phase contrast ; the nucleolus is clearly visible. $a^{\prime \prime}:$ merge of the figures a and a'; $b$ - Mitotic HeLa cells labeled with antiUBF antibodies. The localization of UBF as 3 spots associated with chromosomes. $b$ ' same cells stained by Dapi to reveal the chromosomes. $b$ '”: merge of the figures a and a'. Bar: $1 \mu \mathrm{m}$
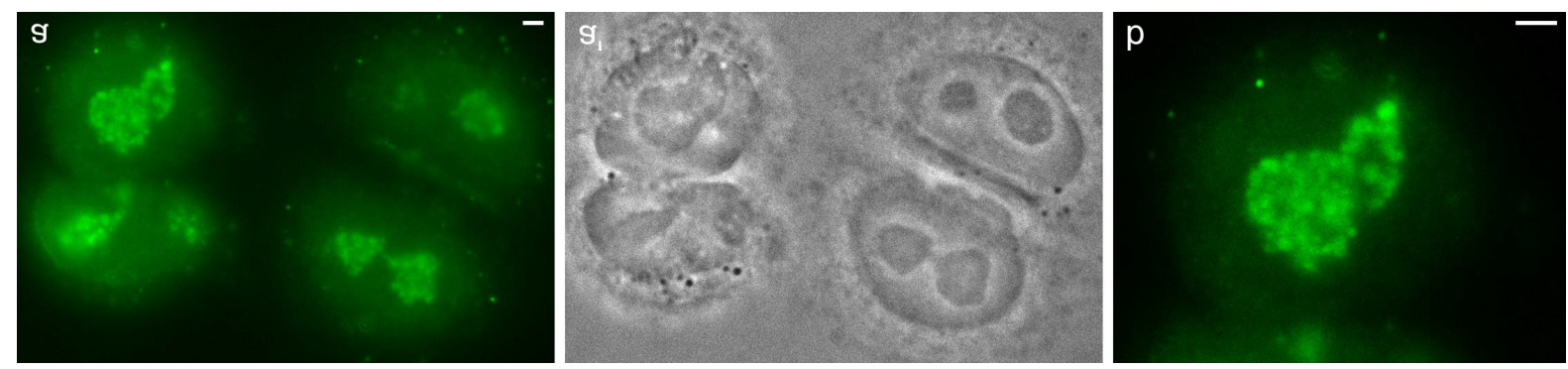

Fig. 4. Localization of dense fibrillar component (DFC). $a$ - Interphasic HeLa cells labeled with anti-fibrillarin antibodies. The localization of fibrillarin is visible in green (FITC) as a network in the central regions of the nucleolus. $a$ ' phase contrast of the same field as figure a. $a$ "' merge of the figures a and a'. Bar: $1 \mu \mathrm{m}$

\section{Modifications of nucleolar organization}

A - Structural and functional nucleolar disorganization after genetic modifications of the tandem organization of yeast rDNA has been described. Nucleologenesis and nucleolar organization analyzed by EM in yeast strains in which tandemly repeated rDNA was replaced by the equivalent number of plasmids bearing single rDNA repeats, demonstrated that the nucleolar structure is modified but still divided into functional subcompartments as is the wild type nucleolus (Trumtel et al. 2000). Upon repression of rDNA transcription in an RNA polymerase I (pol I) thermosensitive mutant, the nucleolar substructure falls apart indicating that rRNA is a primary 
determinant for the assembly of the nucleolus (Trumtel et al. 2000). By deletion or mutation of factors interacting with rDNA (Sir2 and Net1 respectively), it was demonstrated that the distribution of rDNA clusters can regulate nucleolar structure and activity (Shou et al. 2001). This supports the hypothesis that the conformation of the rDNA in clusters is necessary to maintain the nucleolar organization as a cooperative functional domain, and that modifications of rDNA conformation would induce global nucleolar structural defects.

B - Specific inhibition of rDNA transcription can be induced by drugs such as AMD. Early observations showed that low doses of AMD preferentially inhibit rRNA synthesis in cultured animal cells, an effect due to binding of the drug to the rDNA.

The segregation of nucleoli observed in these conditions is characterized by the separation of the nucleolar components that remain superimposed but no longer intermingle (Fig. 2a) (for reviews see (Hadjiolov 1985, Puvion-Dutilleul et al. 1997)). After a brief AMD treatment, the 5' external transcribed sequences (ETS) are associated with the border of the FC and limited areas of the DFC. During a complete block of rRNA transcription with high doses of AMD no labeling is detectable in agreement with the complete absence of transcription and the continued processing of preformed pre-rRNAs (Puvion-Dutilleul et al. 1997).

Transcription of the rRNA genes may also be selectively blocked by microinjection of antibodies against RNA pol I (Scheer and Benavente 1990). Shortly after injection, the DFC starts to split and to be released from the nucleolus until the entire nucleoplasm is filled with numerous extranucleolar fragments. These appear by EM as aggregates composed of fibrillar material. Thus, it was concluded that the DFC is critically dependent on ongoing transcription of the rDNA

C - The adenosine analogue, 5,6 dichloro-1-ribofuranosylbenzimidazole (DRB) has a repressive effect on RNA pol II transcription. However, DRB does not inhibit RNA pol I transcription (Granick 1975a), and induces unraveling of nucleoli into necklace structures (Granick 1975b). In situ hybridization with a specific rDNA probe revealed the unraveling and the 3-D dispersion of most of the rDNA (Haaf and Ward 1996, Junéra et al. 1997).
RNA pol I was detected in each bead of the necklace (Scheer et al. 1984) as was also the transcription factor UBF (Le Panse et al. 1999). Conversely, the nucleolar proteins involved in rRNA processing are mislocalized in large bodies (our unpublished data) derived from the GC. These observations support the hypothesis that the activity of the processing machinery also plays an essential role in the maintenance of the nucleolus. It was also found that inhibitors of cycline-dependent kinases $(\mathrm{CDK})$ as roscovitine can disconnect transcription from the processing machinery (Sirri et al. 2002). EM observations showed that the nucleolar components are dispersed in the nucleoplasm (Fig. 2b).

In addition, there are several examples demonstrating that overexpression of nucleolar proteins can alter nucleolar organization. This indicates that there is an equilibrium between the partners of the nucleolar machineries and that the nucleolar organization reflects the steady state and cooperation of the different processes necessary to build a ribosome.

\section{MACROMOLECULAR DISASSEMBLY OF NUCLEOLI DURING MITOSIS}

In higher eukaryotic cells, nucleolar assembly takes place around the NORs when cells exit from mitosis, and coincides with the reactivation of rDNA transcription. Conversely nucleolar breakdown occurs in prophase concomitantly with repression of rDNA transcription and compaction of the interphase chromatin into mitotic chromosomes.

\section{Release of the nucleolar processing machinery in prophase}

At the end of G2, when the compaction of chromatin into chromosomes can be detected at the nuclear periphery while the nuclear envelope is still present, the shape of the nucleolus is modified. This is due to the progressive release of the processing machineries from the nucleolus. Indeed, while chromatin condenses into chromosomes during prophase, relocalization of the rRNA processing factors occurs and these nucleolar machineries translocate to the chromosome periphery forming a perichromosomal compartment (Fig. 6).. 


\section{Nucleolus}
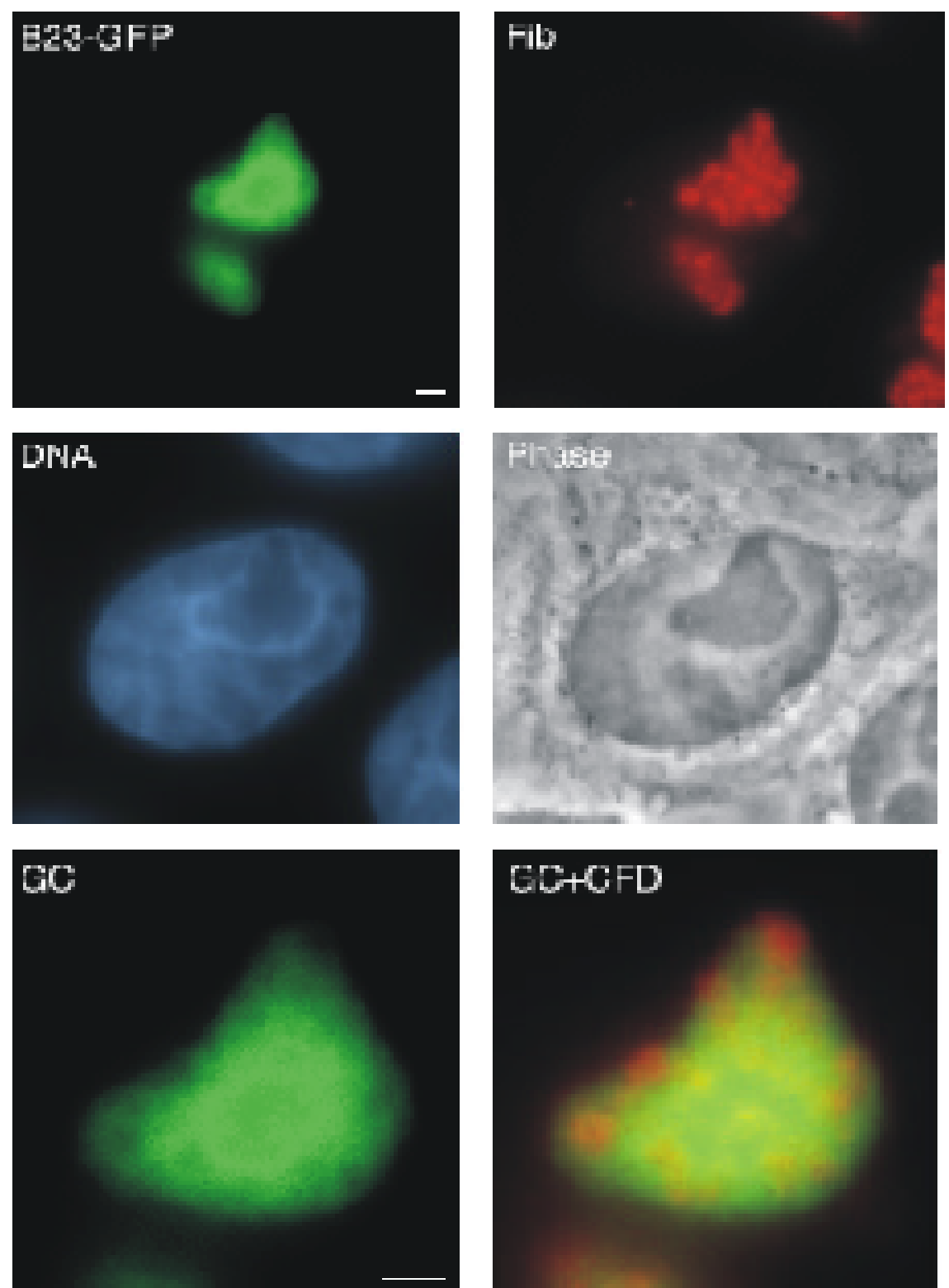

ig. 5. Localization of granular component (GC) and dense fibrillar component (DFC). Interphasic HeLa cells expressing protein B23 tagged with the green fluorescent protein (GFP) that is labeled with anti-fibrillarin antibodies (red). The localization of B23-GFP is visible in the GC in green whereas the fibrillarin forms a network in the central regions of the nucleolus. Phase contrast of the cell shows the nucleolus and Dapi staining and the chromatin around the nucleolus. High magnification of the labeling of the GC and DFC. Bar: $1 \mu \mathrm{m}$
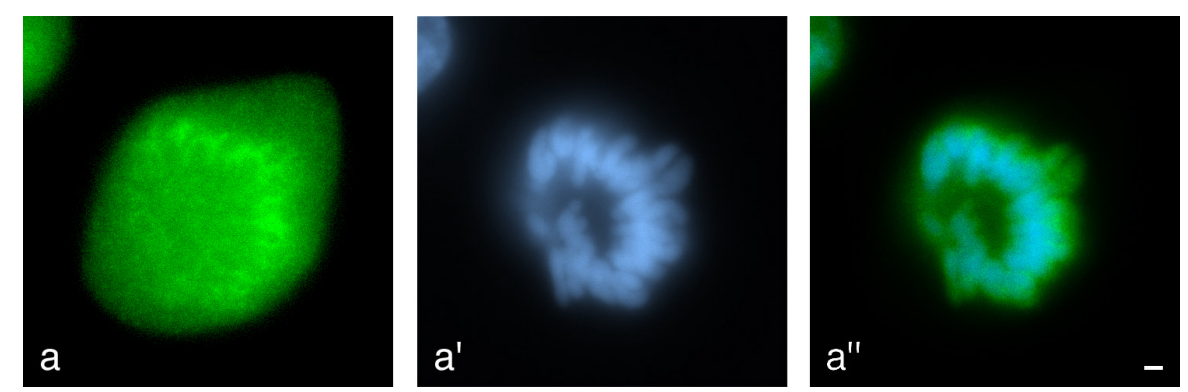

Fig. 6. Localization of GC proteins around the chromosome during mitosis. $a$ - Mitotic HeLa cells labeled with B23 antibodies. The localization of B23 is visible in green (FITC) as a network in the central regions of the cell. $a$ ' phase contrast of the same field as figure a. $a$ " merge of the figures a and a' showing that the green labeling surrounds the chromosomes. Bar: $1 \mu \mathrm{m}$ 
This behavior of the nucleolar processing proteins at the entry of mitosis is general, since it was observed in several mammalian (human, marsupial, hamster, mouse) cells, and in Xenopus and plant cells. The nucleolar proteins that relocate to the chromosome periphery belong to the DFC and $\mathrm{GC}$ of the active nucleolus. Fibrillarin in association with U3 snoRNAs (in DFC) as well as protein B23 and Nop52 (in GC) are the models of these nucleolar proteins (Fomproix et al. 1998, Gautier et al. 1994). In addition, other nucleolar proteins behave similarly (for example nucleolin, PM-Scl 100 and $\mathrm{Ki}$ 67) supporting the view that proteins from the DFC and GC most frequently translocate from the nucleolus to the chromosome periphery during mitosis.

The mechanism controlling the translocation around chromosomes is presently unknown as is the relationship between the nucleolar proteins and chromosomes. However, because entry into the mitotic phase coincides with specific phosphorylation of nucleolin and B23 by the CDK1 kinase, it has been suggested that this phosphorylation controls mitotic changes in nucleolar protein localization and activity. If true, this would mean that nucleolar dispersion is controlled by the same mechanisms as chromosome condensation, spindle formation and nuclear envelope breakdown

\section{Repression of rDNA transcription in prophase}

When cells enter into mitosis, rDNA transcription is detected in prophase (Gébrane-Younès et al. 1997). Quantitative analyses at different periods of mitosis, indicate that nucleolar transcription decreases by about $30 \%$ during early prophase compared to interphase and stops in late prophase (Prescott and Bender 1962). Although the timing of pol I transcription arrest during prophase remains to be precisely determined, it most probably occurs concomitantly or shortly before nuclear envelope breakdown. After breakdown, the nucleolus is no longer visible among the condensed chromosomes

\section{Partly processed rRNA generated at the beginning of mitosis}

In prophase, the rRNA processing machinery is released from the nucleolus before total repression of rDNA transcription. The rRNAs synthesized at this period accumulate as partially processed $45 \mathrm{~S}$ pre-rRNAs detected by ETS probes (Dousset et al. 2000). This confirms previous observations that $45 \mathrm{~S}$ and $32 \mathrm{~S}$ rRNAs are present in mitotic cells even in metaphase-arrested cells (Fan and Penman 1971), and also the hypothesis that RNA contained at the chromosome periphery is synthesized at the end of G2 or at the beginning of prophase (Moyne and Garrido 1976). In prophase, these pre-rRNAs are localized in the nucleoli whereas after nucleolar disassembly they are at the chromosome periphery, and also in the cytoplasm from prometaphase to telophase. After prophase, no significant signal for the presence of pre-RNAs has been found at the NORs, supporting the suggestion that these prerRNAs are released from the template (Beven et al. 1996, Dundr and Olson 1998, Weisenberger and Scheer 1995). These partially processed pre-rRNAs are colocalized with the rRNA processing proteins at the chromosome periphery and also in nucleolarderived foci observed in the cytoplasm of mitotic cells. This co-localization is consistent with the fact that ribonucleoprotein complexes containing both pre-rRNAs and processing proteins are isolated from mitotic cells.

In conclusion, nucleolar breakdown occurs in prophase concomitantly with chromatin compaction into mitotic chromosomes. It is a two step process involving first the translocation of the processing machineries around the chromosomes while transcription of rDNA is still active, followed by repression of rDNA transcription. Consequently, partly processed rRNAs are generated during prophase. We presently do not know how disassembly of the processing machineries from the rDNA transcription sites in prophase is regulated and controlled.

\section{Pol I transcription machinery remains assembled in a repressed form during mitosis}

From prometaphase to anaphase the rDNA transcription activity is abolished. However, even in the absence of transcription, the components of the rDNA transcription machinery are associated with rDNA (Sirri et al. 1999). It was proposed that the inhibition of mitotic RNA pol I transcription was caused by phosphorylation of components of the rDNA transcription machinery directed by CDK1-cyclin B (Heix et al. 1998, Sirri et al. 2000). The role of CDK1-cyclin B in mitotic repression of rDNA transcription was clearly demonstrated by inhibiting the CDK1-cyclin B kinase pathway in mitotic cells. Indeed, inhibition of this pathway in mitotic cells induces resumption of rDNA transcription (Sirri et al. 2000).

In human cells there are 10 NOR-bearing chromosomes, but the rDNA transcription machinery remains associated in variable amounts with only six of them, at least in HeLa cells (Roussel et al. 1996). It is interesting to note that the amount of proteins associated with both chromatids of the same 
chromosome appears equivalent. Consequently, the separation of chromatids occurring during anaphase leads to the equal partition of the rDNA transcription machinery and therefore between the two daughter cells.

\section{Distribution of the processing machinery around the chromosome periphery during mitosis is CDK1-dependent}

At the chromosome periphery the nucleolar processing complexes are regularly distributed without visible foci, using immunofluorescence labeling. The number of complexes at the chromosome periphery progressively increases during anaphase and telophase. This suggests a cellcycle related behavior. Indeed, inhibition of CDK1 in mitotic-arrested cells induces relocation of the nucleolar processing machineries (Sirri et al. 2002) demonstrating that location at the chromosome periphery is controlled during mitosis by the CDK1 pathway. Inhibition of CDK1 induces the formation of foci corresponding to prenucleolar bodies (PNBs) and only a partial translocation of the processing machinery to transcription sites. Accordingly, there is no rRNA cleavage of the new transcripts synthesized in mitotic-arrested cells (Sirri et al. 2002). Part of the early processing machinery is efficiently recruited on these pre-rRNAs indicating that the CDK1 pathway regulates this recruitment. As methylation and pseudouridylation of these large rRNAs are not documented, we do not know if the CDK1 pathway is also involved in the control of these rRNA modifications. Noticeably, proteins and snoRNAs involved in rRNA processing are observed in PNBs, before localizing at sites containing newly transcribed rRNAs (Jiménez-Garcia et al . 1994). It was proposed that PNBs are mobile nuclear bodies that participate in the delivery of the rRNAprocessing complexes to sites of rDNA transcription (Jiménez-Garcia et al. 1994).

\section{MACROMOLECULAR ASSEMBLY OF NUCLEOLI AT EXIT OF MITOSIS}

Nucleolar assembly occurs when cells exit from mitosis. Reports suggest that nucleolar assembly depends on the activation of the pol I transcription machinery (Scheer and Hock 1999, Thiry and Goessens 1996). This generates pre-rRNAs (47S in mammals), that recruit the rRNA-processing machinery. Noticeably, proteins and snoRNAs involved in rRNA processing are observed in PNBs, before localizing at sites containing newly transcribed rRNAs.
Activation of transcription in telophase

At telophase, the resumption of rDNA transcription occurs simultaneously in each pol I transcription machinery-associated NOR and the level of rDNA transcription activity seems to be directly related to the amount of pol I machinery present in the NOR (Roussel et al. 1996). In HeLa cells, the activation of six NORs induces six individual foci of pol I transcription. From EM investigations, we know that the site of this initial transcription of rDNA is located close to the reforming nuclear envelope (Hernandez-Verdun et al. 1980). Recently, we have also observed in living cells that the NORs, imaged by fibrillarin recruitment at the initial stage of nucleolar building, are distributed in the nucleus in distinct foci moving slowly within the nuclear volume without apparent coordination (Savino et al. 2001).

The CDK1-cyclin B kinase activity maintains mitotic repression of rDNA transcription. Indeed, inhibition of the CDK1-cyclin B kinase pathway in mitotic cells induces resumption of rDNA transcription. We propose that inactivation of the CDK1-cyclin B kinase occurring normally in telophase is sufficient to release mitotic repression of rDNA transcription while this is not sufficient to restore proper nucleolar assembly (Sirri et al. 2002). Relocation of the processing machinery

In living cells, the processing nucleolar proteins tagged with GFP such as fibrillarin-GFP and Nop52-GFP, are concentrated around the chromosomes during anaphase. In telophase, the tagged proteins concentrate in many foci, corresponding to PNBs. We recently demonstrated that the PNBs form on the chromosome surface and remain associated with condensed chromatin. Strikingly, fibrillarin concentrates in PNBs and NORs when a decrease in CDK1 activity overcomes the mitotic repression of RNA pol I transcription, while Nop52s appear progressively recruited in NORs. Thus, it seems that recruitment of the processing machinery at the time of nucleolar assembly is a regulated process probably depending on cell cycle progression.

Based on observations using fixed cells, the prediction was that the PNBs move to deliver preassembled processing complexes to the site of rDNA transcription. PNB dynamics in living cells do not reveal such directed movement towards the nucleolus (Dundr et al. 2000, Savino et al. 2001). Rather, the progressive delivery of PNB proteins to the nucleoli is ensured by directional flow between PNBs and between PNBs and the nucleolus. The role of this intricate delivery pathway remains an open question, in particular if it is cell cycle controlled. Indeed, the dynamics of fibrillarin and 
other processing proteins analyzed by fluorescence recovery after photobleaching indicate rapid diffusion in the nucleoplasm and permanent recruitment in the nucleolus (Phair and Misteli 2000). However, the mobile fraction can be different in nucleoli and Cajal (Coiled) bodies (Snaar et al. 2000 ) indicating that the residence time depends on specific interactions (Misteli 2001). Therefore the formation of PNBs at the mitosis/interphase transition suggests a steady state at this period of the cell cycle, favoring residence of processing factors close to the condensed chromatin either by selfassembly of processing factors or by specific interaction with pre-rRNAs.

\section{Role of partly processed rRNA in nucleolar assembly}

At the mitosis/interphase transition, the processing complexes forming PNBs can be nucleated by prerRNAs passing through mitosis (Dousset et al. 2000, Dundr et al. 2000, Dundr and Olson 1998, PinolRoma 1999). This scenario is based on the fact that pre-rRNAs are localized in PNBs (Dousset et al. 2000) and the pre-RNAs as well as rRNA processing intermediates are immunopurified in mitotic processing complexes (Pinol-Roma 1999). This model of PNB formation by association around pre-rRNAs could explain the temporal order of nucleolar delivery of the processing machinery driven by pre-rRNA stability. Self-association of processing proteins in PNBs cannot be excluded because PNBs are generated without any rRNAs in reconstituted nuclei in Xenopus extracts (Bell et al. 1992, Verheggen et al. 2000). However, during de novo nucleolar assembly in Xenopus embryos prior to activation of zygotic transcription, the presence of pre-rRNAs was demonstrated in association with regrouping of PNBs around NORs (Verheggen et al. 2000).

In conclusion, the recruitment of the nucleolar processing machinery during nuclear assembly in early G1 involves the formation of PNBs which is either an assembly or a storage platform of processing complexes associated with pre-rRNAs. The stability of these PNBs seems to be cell cycle regulated.

\section{CONCLUSIONS}

As proposed by Mélèse (Mélèse and Xue 1995), the nucleolus is a "functional domain formed by the act of building a ribosome". The steady state between transcription, processing and export of ribosomal subunits generates this large domain, the nucleolus present during interphase. During nucleolar assembly or disassembly, this steady state is different since the pol I machinery remains assembled with rDNA in a repressed form whereas the processing machineries are delocalized to the chromosome periphery. Recent findings indicate that nucleolar assembly at exit from mitosis depends on cell cycle controls. Indeed, CDK1 activity represses pol I transcription during mitosis, and its inactivation releases this silencing. The formation of PNBs is also controlled by the CDK1 pathway, whereas the recruitment of the rRNA-processing machinery appears to depend on the activity of another CDK. The characterization of the CDK(s) controlling this process should be investigated in the future. Ribosome biogenesis involves the pol-I, polII and pol-III-dependent transcription pathways, the translocation of 5S RNAs and the ordered assembly of ribosomal proteins. Presently we do not know how these pathways are coordinated during assembly or disassembly of the nucleolus. This should therefore be an important goal of future research.

The pre-rRNAs generated by pol I transcription are localized at the sites of active rDNA gene clusters. The binding affinity of the processing proteins for these pre-rRNAs can explain the compartmentalization of the processing machinery in the functional nucleolus. During nucleolar assembly, pre-rRNAs also appear to participate in compartmentalization of the processing machinery. Mitotic pre-rRNAs are involved in the reformation of the nucleolus after mitosis, and maternal prerRNAs in Xenopus embryos are involved in regrouping PNBs around the rDNA. In both situations, an intriguing question is how the mitotic pre-rRNAs or the maternal pre-rRNAs regroup around the rDNA genes. The presence of pre-rRNAs in PNBs could also explain the formation of temporarily organized bodies. The stability of these rRNAs could determine their lifetime. Clearly, these questions must be addressed if we are to understand the role of stable rRNAs in the formation and/or maintenance of nuclear structures.

Another interesting and promising field of research is how the presence of a functional nucleolus contributes to the general nuclear architecture. It has been recently reported that the loci at the nucleolar periphery are significantly less mobile than the others and disruption of nucleoli increase the mobility of nucleolar-associated loci (Chubb et al. 2002). This indicates that chromatin associated with the nucleolus is more restricted in its movements than non-associated chromatin. It is interesting to note that the nucleolus is the first active nuclear domain to be assembled at the end of mitosis, it creates a domain of sequestration or exclusion of molecules participating in cellular 
functions different from ribosome biogenesis. The exclusion of pol II from the nucleolus could explain why the nucleolus is the site of silencing for inserted pol II genes. However we do not know how this exclusion propriety is generated and when during assembly of nucleolar functions it occurs.

Last but not least, the inventory of the proteins found in the isolated nucleolus by mass spectrometry has opened a new field of research (Andersen et al. 2002). A total of 271 nucleolar proteins were initially identified among which were 80 novel proteins. Functional classification of these proteins is now in progress to determine what proteins are involved in ribosome production and what proteins are dedicated to other functions (Scherl et al. 2002). This global approach provides convincing evidence of the role of the nucleolus in the control of gene expression.

\section{ACKNOWLEDGEMENTS}

The authors thank Isabelle Berthuy for the immunolocalizations, Myriam Barre for help with the photographic work and A.L. Haenni for critical reading of the manuscript. This work was supported in part by grants from the Centre National de la Recherche Scientifique (UMR 7592) and the Association pour la Recherche sur le Cancer (Contracts 5304 and 4290).

Simultaneously published in J. Berger (ed): Advances in Cell Biology. Kopp Publ., České Budějovice 2003, pp. 9-38.

\section{REFERENCES}

Andersen J.S., C.E. Lyon, A.H. Fox, A.K.L. Leung, Y.W. Lam, H. Steen, M. Mann, A. I. Lamond: Directed proteomic analysis of the human nucleolus. Curr. Biol. 12: 1-11, 2002.

Bell P., M.C. Dabauvalle, U. Scheer: In vitro assembly of prenucleolar bodies in Xenopus egg extract. J. Cell Biol. 118: 1297-1304, 1992.

Beven A.F., R. Lee, M. Razaz, D.J. Leader, J.W.S. Brown, P.J. Shaw: The organization of ribosomal RNA processing correlates with the distribution of nucleolar snRNAs. J. Cell Sci. 109: 1241-1251, 1996.

Biggiogera M., M. Malatesta, S. AbolhassaniDadras, F. Amalric, L.I. Rothblum, S. Fakan: Revealing the unseen: the organizer of the nucleolus. J. Cell Sci. 17: 3199-3205, 2001.
Busch H. and K. Smetana: The nucleus of cancer cells. In H. Busch (ed), The Molecular Biology of Cancer. Academic Press, New York 1974, pp. 41-80.

Carmo-Fonseca M., L. Mendes-Soares, I. Campos: To be or not to be in the nucleolus. Nature Cell Biol. 2: 107-112, 2000.

Chubb J.R., S. Boyle, P. Perry, W.A. Bickmore: Chromatin motion is constrained by association with nuclear compartments in human cells. Curr. Biol. 12: 439-445, 2002.

Cmarko D., P.J. Verschure, L.I. Rothblum, D. Hernandez-Verdun, F. Amalric, R. van Driel, S. Fakan: Ultrastructural analysis of nucleolar transcription in cells microinjected with 5-bromo-UTP. Histochem. Cell Biol. 113: 181187, 2000.

Dousset T., C. Wang, C. Verheggen, D. Chen, D. Hernandez-Verdun, S. Huang: Initiation of nucleolar assembly is independent of RNA polymerase I transcription. Mol. Biol. Cell 11: 2705-2717, 2000.

Dundr M., T. Misteli, M.O.J. Olson: The dynamics of postmitotic reassembly of the nucleolus. J. Cell Biol. 150: 433-446, 2000.

Dundr M. and M.O.J. Olson: Partially processed pre-rRNA is preserved in association with processing components in nucleolus derived foci during mitosis. Mol. Biol. Cell 9: 2407-2422, 1998.

Fan H. and S. Penman: Regulation of synthesis and processing of nucleolar components in metaphase-arrested cells. J. Mol. Biol. 59: 2742, 1971.

Fatica A. and D. Tollervey: Making ribosomes. Curr. Opin. Cell Biol. 14: 313-318, 2002.

Fomproix N., J. Gébrane-Younes, D. HernandezVerdun: Effects of anti-fibrillarin antibodies on building of functional nucleoli at the end of mitosis. J. Cell Sci. 111: 359-372, 1998.

Gautier T., N. Fomproix, C. Masson, M.C. AzumGélade, N. Gas, D. Hernandez-Verdun: Fate of specific nucleolar perichromosomal proteins during mitosis: Cellular distribution and association with U3 snoRNA. Biol. Cell 82: 8193, 1994.

Gébrane-Younès J., N. Fomproix, D. HernandezVerdun: When rDNA transcription is arrested during mitosis, UBF is still associated with noncondensed rDNA. J. Cell Sci. 110: 2429-2440, 1997.

Gerlich D., J. Beaudouin, B. Kalbfuss, N. Daigle, R. Eils, J. Ellenberg: Global chromosome positions are transmitted through mitosis in mammalian cells. Cell 112: 751, 2003.

Goessens G.: Nucleolar structure. Int. Rev. Cytol. 87: 107-158, 1984. 
Granick D.: Nucleolar necklaces in chick embryo fibroblast cells. I. Formation of necklaces by dichlororibobenzimidazole and other adenosine analogues that decrease RNA synthesis and degrade preribosomes. J. Cell Biol. 65: 398-417, 1975a.

Granick D.: Nucleolar necklaces in chick embryo fibroblast cells. II. Microscope observations of the effect of adenosine analogues on nucleolar necklace formation. J. Cell Biol. 65: 418-427, $1975 b$.

Haaf T. and D.C. Ward: Inhibition of RNA polymerase II transcription causes chromatin decondensation, loss of nucleolar structure, and dispersion of chromosomal domains. Exp. Cell Res. 224: 163-173, 1996.

Hadjiolov A.A.: The nucleolus and ribosome biogenesis. In M. Alfert, W. Beermann, L. Goldstein, K.R. Porter, P. Sitte, (eds) : Cell Biology Monographs, Vol. 12, Springer-Verlag, Wien 1985.

Harnpicharnchai P., J. Jakovljevic, E. Horsey, T. Miles, J. Roman, M. Rout, D. Meagher, B. Imai, Y. Guo, C.J. Brame, J. Shabanowitz, D.F. Hunt, J.L. Woolford: Composition and functional characterization of yeast $66 \mathrm{~S}$ ribosome assembly intermediates. Mol. Cell 8: 505-515, 2001.

Heix J., A. Vente, R. Voit, A. Budde, T.M. Michaelidis, I. Grummt: Mitotic silencing of human rRNA synthesis: inactivation of the promoter selectivity factor SL1 by cdc2/cyclin B-mediated phosphorylation. EMBO J. 17: 7373-7381, 1998.

Hernandez-Verdun D., C.A. Bourgeois, M. Bouteille: Simultaneous nucleologenesis in daughter cells during late telophase. Bio Cell 37: $1-4,1980$.

Hozak P., J.T. Novak, K. Smetana: Threedimensional reconstructions of nucleolusorganizing regions in PHA-stimulated human lymphocytes. Biol. Cell 66: 225-233, 1989.

Jiménez-Garcia L.F., M. de L. Segura-Valdez, R.L Ochs, L.I. Rothblum, R. Hannan, D.L. Spector: Nucleologenesis: U3 snRNAcontaining prenucleolar bodies move to sites of active pre-rRNA transcription after mitosis. Mol. Biol. Cell 5: 955-966, 1994.

Junéra H. R., C. Masson, G. Géraud, D. HernandezVerdun: The three-dimensional organization of ribosomal genes and the architecture of the nucleoli vary with G1, S and G2 phases. J. Cell Sci. 108: 3427-3441, 1995.

Junéra H.R., C. Masson, G. Géraud, J. Suja, D. Hernandez-Verdun: Involvement of in situ conformation of ribosomal genes and selective distribution of UBF in rRNA transcription. Mol. Biol. Cell 8: 145-156, 1997.
Le Panse S., C. Masson, L. Héliot, J.-M. Chassery, H. R. Junéra, D. Hernandez-Verdun: 3-D organization of single ribosomal transcription units after DRB inhibition of RNA polymerase II transcription. J. Cell Sci. 112: 2145-2154, 1999.

Leung A.K.L. and A.I. Lamond: In vivo analysis of NHPX reveals a novel nucleolar localization pathway involving a transient accumulation in splicing speckles. J. Cell Biol. 157: 615-629, 2002.

McClintock B.: The relation of particular chromosomal element to the development of the nucleoli in Zea mays. Z. Zellforsch. mikrosk. Anat. 21: 294-328, 1934.

Mélèse T. and Z. Xue: The nucleolus: an organelle formed by ,the act of buiding a ribosome. Curr. Opin. Cell Biol. 7: 319-324, 1995.

Misteli T.: Protein dynamics: implications for nuclear architecture and gene expression. Science 291: 843-847, 2001.

Moyne G. and J. Garrido: Ultrastructural evidence of mitotic perichromosomal ribonucleoproteins in hamster cells. Exp. Cell Res. 98: 237-247, 1976.

Nissan T.A., J. Bassler, E. Petfalski, D. Tollervey, E. Hurt: $60 \mathrm{~S}$ pre-ribosome formation viewed from assembly in the nucleolus until export to the cytoplasm. EMBO J. 21: 5539-5547, 2002.

Olson M.O.J., M. Dundr, A. Szebeni: The nucleolus: an old factory with unexpected capabilities. Trends Cell Biol. 10: 189-196, 2000.

Pébusque M.J. and R. Seïte: Electron microscopic studies of silver-stained proteins in nucleolar organizer regions: location in nucleoli of rat sympathetic neurons during light and dark periods. J. Cell Sci. 51: 85-94, 1981.

Pederson T.: The plurifunctional nucleolus. Nucl. Acids Res. 26: 3871-3876, 1998.

Phair R.D., and T. Misteli: High mobility of proteins in the mammalian cell nucleus. Nature 404: 604-609, 2000.

Pinol-Roma S.: Association of nonribosomal nucleolar proteins in ribonucleoprotein complexes during interphase and mitosis. Mol. Biol. Cell 10: 77-90, 1999.

Prescott D.M. and M.A. Bender : Synthesis of RNA and protein during mitosis in mammalian tissue culture cells. Exp. Cell Res. 26: 260-268, 1962.

Puvion-Dutilleul F., J.-P. Bachellerie, E. Puvion Nucleolar organization of HeLa cells as studied by in situ hybridization. Chromosoma 100: 395409, 1991.

Puvion-Dutilleul F., E. Puvion, J.-P. Bachellerie: Early stages of pre-rRNA formation within the nucleolar ultrastructure of mouse cells studied by in situ hybridization with 5'ETS leader probe. Chromosoma 105: 496-505, 1997. 
Roussel P., C. André, L. Comai, D. HernandezVerdun: The rDNA transcription machinery is assembled during mitosis in active NORs and absent in inactive NORs. J. Cell Biol. 133: 235246, 1996.

Savino T.M., J. Gébrane-Younès, J. De Mey, J.-B. Sibarita, D. Hernandez-Verdun: Nucleolar assembly of the rRNA processing machinery in living cells. J. Cell Biol. 153: 1097-1110, 2001.

Scheer U. and R. Benavente: Functional and dynamic aspects of the mammalian nucleolus. BioEssays 12: 14-21, 1990.

Scheer U. and R. Hock: Structure and function of the nucleolus. Curr. Opin. Cell Biol. 11: 385390, 1999.

Scheer U., B. Hügle, R. Hazan, K.M. Rose: Druginduced dispersal of transcribed rRNA genes and transcriptional products: immunolocalization and silver staining of different nucleolar components in rat cells treated with 5,6-dichloro-ß-Dribofuranosylbenzimidazole. J. Cell Biol. 99: 672-679, 1984.

Scherl A., Y. Couté, C. Déon, A. Callé, K. Kindbeiter, J.-C.Sanchez, A. Greco, D. Hochstrasser, J.-J. Diaz: Functional proteomic analysis of human nucleolus. Molec. Biol. Cell 13: 4100-4109, 2002.

Shaw P.J. and E.G. Jordan: The nucleolus. Annu. Rev. Cell Dev. Biol. 11: 93-121, 1995.

Shou W., K.M. Sakamoto, J. Keener, K.W. Morimoto, E.E.Traverso, R. Azzam, G.J. Hoppe, R.M. Feldman, J.DeModena, D. Moazed, H. Charbonneau, M. Nomura, R..J. Deshaies: Net1 stimulates RNA polymerase I transcription and regulates nucleolar structure independently of controlling mitotic exit. Mol. Cell 8: 45-55, 2001.

Sirri V., D. Hernandez-Verdun, P. Roussel: Cyclin-dependent kinases govern formation and maintenance of the nucleolus. J. Cell Biol. 156: 969-981, 2002.

Sirri V., P. Roussel, D. Hernandez-Verdun: The mitotically phosphorylated form of the transcription termination factor TTF-1 is associated with the repressed rDNA transcription machinery. J. Cell Sci. 112: 3259-3268, 1999.

Sirri V., P. Roussel, D. Hernandez-Verdun: In vivo release of mitotic silencing of ribosomal gene transcription does not give rise to precursor ribosomal RNA processing. J. Cell Biol. 148: 259-270, 2000.

Snaar S., K. Wiesmeijer, A.G. Jochemsen, H.J. Tanke, R.W.Dirks: Mutational analysis of fibrillarin and its mobility in living human cells. J. Cell Biol. 151: 653-662, 2000.

Thiry M. and G. Goessens: The nucleolus during the cell cycle. In Molecular Biology Intelligence Unit. Springer-Verlag, Heidelberg 1996.

Thiry M. and L. Thiry-Blaise: Locating transcribed and non-transcribed rDNA spacer sequences within the nucleolus by in situ hybridization and immunoelectron microscopy. Nucleic Acids. Res. 19: 11-15, 1991.

Trumtel S., I. Léger-Silvestre, P.-E. Gleizes, F. Teulières, N. Gas: Assembly and functional organization of the nucleolus: ultrastructural analysis of Saccharomyces cerevisiae mutants. Mol. Biol. Cell 11: 2175-2189, 2000.

Tsai R.Y.L. and R.D.G. McKay: A nucleolar mechanism controlling cell proliferation in stem cells and cancer cells. Genes dev. 16: 29913003, 2002.

Verheggen C., G. Almouzni, and D. HernandezVerdun: The ribosomal RNA processing machinery is recruited to the nucleolar domain before RNA polymerase I during Xenopus laevis development. J. Cell Biol. 149: 293-305, 2000.

Visintin R. and A. Amon: The nucleolus: the magician's hat for cell cycle tricks. Curr. Opin. Cell Biol. 12: 372-377, 2000.

Weisenberger D. and U. Scheer: A possible mechanism for the inhibition of ribosomal RNA gene transcription during mitosis. J. Cell Biol. 129: 561-575, 1995.

\section{Address:}

Danièle Hernandez-Verdun, Institut Jacques Monod, 2 place Jussieu, 75251 Paris Cedex 05, France 